A Occlusion of Aneurysms by Size

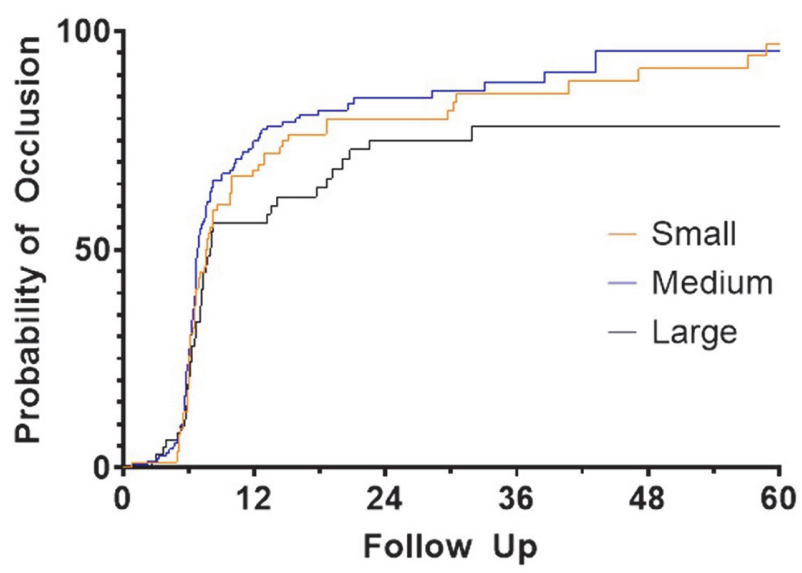

B Occlusion of Aneurysms by \# of PEDs

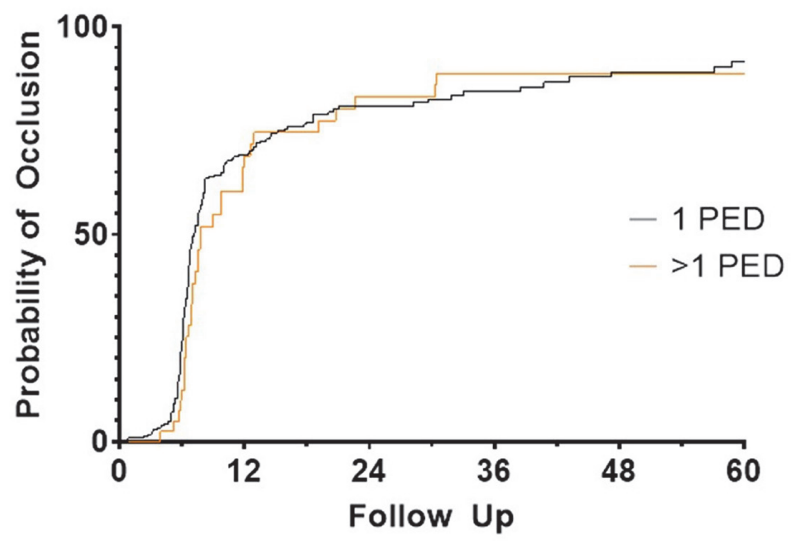

Abstract E-106 Figure 1 (A) Occlusion of Aneurysms by Size; (B) Occlusion of Aneurysms by \# of PEDs

grouped by size (small $<4 \mathrm{~mm}$, medium $4-10 \mathrm{~mm}$, large $>10$ $\mathrm{mm}$ ) or number of PED's deployed (1 vs $>1$ ). Kaplan-Meier curves were compared using a log-rank Mantel-Cox test. Time to occlusion as reported as median $(95 \% \mathrm{CI})$.
Results 291 aneurysms in 222 patients were included in this analysis. Large aneurysm size was significantly associated with a lower probability of complete occlusion at 5 years (figure $1 \mathrm{~A}, \mathrm{p}=0.037)$. Aneurysm size was not significantly associated with time to aneurysm occlusion. Median time to occlusion was similar across sizes: 7.7 months (CI 6.7-9.8) for small aneurysms, 6.9 months (CI 6.6-7.6) for medium aneurysms, and 8.0 months (CI 7.1 -17.7) for large aneurysms. When stratified by number of PEDs deployed, survival curves were not significantly different (figure $1 \mathrm{~B}, \mathrm{p}=0.479$ ). Median time to occlusion was 7.2 months (CI 6.7-7.8) for aneurysms treated with 1 PED and 7.7 months (CI 6.9-11.8) for aneurysms treated with multiple PEDs.

Conclusion Aneurysm size and number of PEDs did not significantly alter aneurysm behavior at early angiographic follow up. However, large aneurysms demonstrated an earlier plateau in their likelihood of complete occlusion. These findings are relevant for determining optimum angiographic follow up schedules and providing anticipatory guidance to patients.

Disclosures S. Cler: None. D. Lauzier: None. A. Kansagra: 2; C; Penumbra, Microvention, iSchemaView.

\section{E-107 VERTEBRAL ARTERY INJURY FROM THE OPERATING ROOM TO THE NEUROENDOVASCULAR SUITE: THE EVOLUTION OF AN ALGORITHM}

G Porto*, M Alshareef, J Wessell, A Spiotta. Neurosurgery, Medical University of South Carolina, Charleston, SC

\subsection{6/neurintsurg-2021-SNIS.202}

Background Iatrogenic vertebral artery injury (VAI) during spine surgery is a rare complication commonly occurring in posterior cervical procedures involving C1 and C2 with potentially catastrophic consequences. Management requires immediate intra-operative action as well as vascular control. Objective: To develop an institutional algorithm for standardizing peri-operative decision-making and endovascular approach to spine injury related iatrogenic VAI.

Methods We performed a retrospective review of 1905 patients undergoing cerebral angiography. Adult patients undergoing a cervical spine operation complicated by VAI were

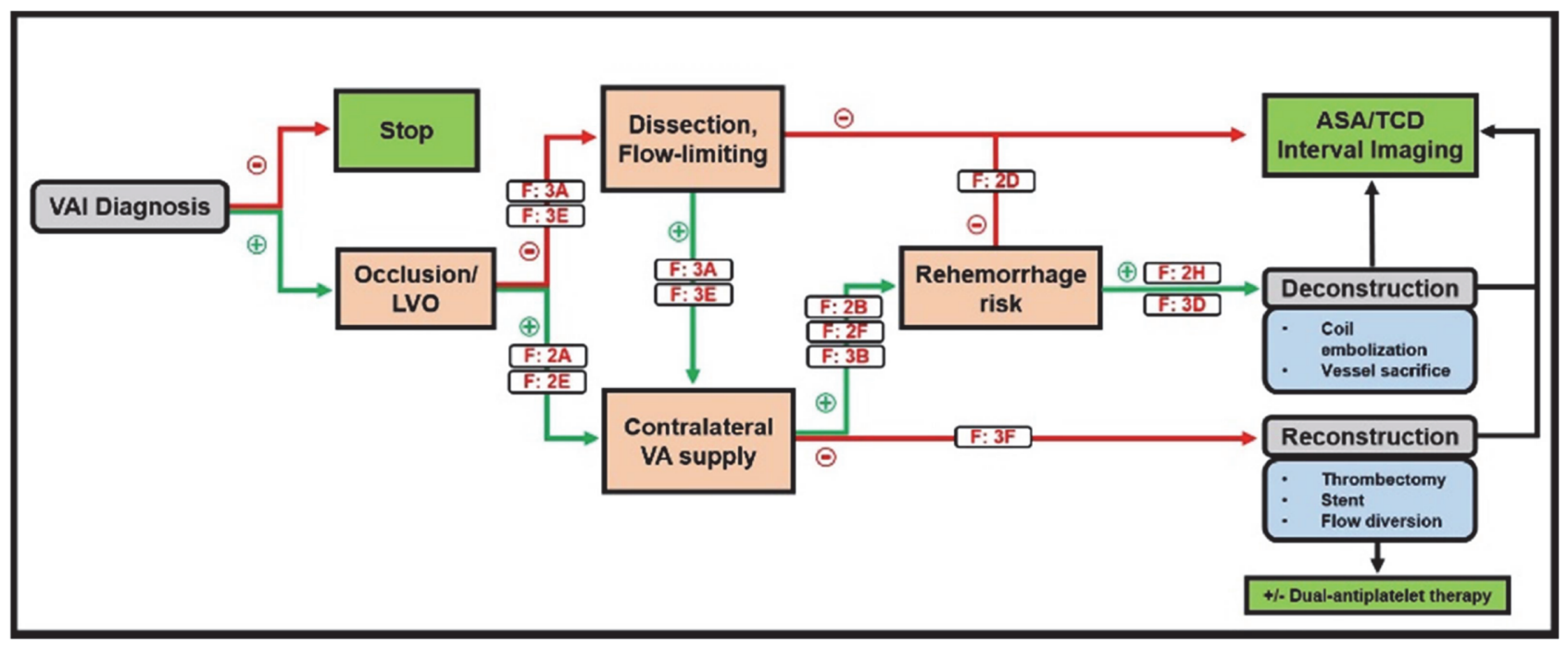


included. Peri-operative variables as well as angiography findings were collected and reviewed.

Results Seven VAI patients were identified with average age of 67 years. Most cases involved the posterior C1, C2 region. Two patients had dominant VAI and five patients had complete occlusion of the vertebral artery. Endovascular treatment was performed in three cases (two cases of coil embolization and one thrombectomy). All cases were reviewed and utilized in the development of an institutional algorithm for spineendovascular decision-making followed by treatment paradigm in the neuroendovascular suite.

Conclusion Iatrogenic VAI is a dreaded complication of spine surgery with oftentimes poor consensus on management strategy. Recognition and rapid management of VAI is vital for mitigation of neurologic injury. We present an institutional algorithm utilizing our single center experience as well as evidence-based literature for rapid endovascular management. We advocate for institution-dependent, collaborative algorithms to assist with such rare yet potentially catastrophic condition.

Disclosures G. Porto: None. M. Alshareef: None. J. Wessell: None. A. Spiotta: None.

\section{E-108 ENDOVASCULAR MANAGEMENT OF DISTAL ANTERIOR CEREBRAL ARTERY ANEURYSMS: A MULTI-CENTER EXPERIENCE}

${ }^{1} \mathrm{G}$ Porto*, 'S Al Kasab, ${ }^{1} \mathrm{M}$ Sattur, ${ }^{2} \mathrm{E}$ Almallouhi, ${ }^{1} \mathrm{O}$ Laithia, ${ }^{1} \mathrm{M}$ Casey, ${ }^{3} \mathrm{R}$ Starke, ${ }^{4} \mathrm{G}$ Lanzino, ${ }^{5} \mathrm{C}$ Ogilvy, ${ }^{5} \mathrm{~A}$ Thomas, ${ }^{6} \mathrm{~L}$ Kim, ${ }^{7} \mathrm{R}$ James, ${ }^{6} \mathrm{M}$ Levitt, ${ }^{1} \mathrm{~A}$ Spiotta. ${ }^{7}$ Neurosurgery, Medical University of South Carolina, Charleston, SC; ${ }^{2}$ Neurology, Medical University of South Carolina, Charleston, SC; ${ }^{3}$ Neurosurgery, University of Miami, Miami, FL; ${ }^{4}$ Neurosurgery, Mayo Clinic, Rochester, MN; ${ }^{5}$ Neurosurgery, Beth Israel Deaconess Medical Center, Boston, MA; ${ }^{6}$ Neurosurgery, University of Washington, Seattle, WA; ${ }^{7}$ Neurosurgery, University of Louisville, Louisville, $K Y$

10.1136/neurintsurg-2021-SNIS.203

Introduction Distal anterior cerebral artery aneurysms (DACAA) are relatively rare, carry high rate of rupture and are often challenging to treat. The evolution of endovascular techniques has allowed for safe and durable treatment of these aneurysms. The purpose of this study is to investigate the safety, efficacy and long-term outcomes of endovascular treatment of DACAA.

Methods Retrospective review of DACAA endovascularly treated at 5 different institutions across the nation. Data collected included baseline demographics, rupture status, radiographic features, endovascular technique, complication rates, and long-term angiographic and clinical outcomes. Primary endpoint was good clinical outcome (modified Rankin scale 02 ). Secondary endpoints included complications and radiographic occlusion at follow up.

Results 84 patients were reviewed (table 1). Mean age was 56, and $64(71.4 \%)$ were female. 52 (61.9\%) aneurysms were ruptured. $60(71.4 \%)$ aneurysms were treated with primary coiling, and the remaining 24 were treated flow diversion. Adequate occlusion was achieved in 41 (95.3\%) aneurysms treated with coiling, and $17(89.5 \%)$ with flow diversion. No patients required retreatment in the flow diversion category versus $16(29 \%)$ in primary coiling. Good functional outcome was achieved in 59 patients (85.5\%), and mortality was observed in $2(2.3 \%)$ patients. There were overall $11(13 \%)$ complications. In primary coiling there were $9(15 \%)$ procedural complications: 5 thromboembolic events, 3 intraoperative ruptures and 1 access site hematoma. In flow diversion
Abstract E-108 Table 1 Patient demographics and aneurysm characteristics

\begin{tabular}{|c|c|}
\hline Age, mean (SD) & $56(14.1)$ \\
\hline Females, n (\%) & $60(71.4 \%)$ \\
\hline \multicolumn{2}{|l|}{ Indication, $\mathbf{n}(\%)$} \\
\hline Subarachnoid hemorrhage & $51(60.7 \%)$ \\
\hline Intraparenchymal hemorrhage & $1(1.2 \%)$ \\
\hline Elective procedure & $32(38.1 \%)$ \\
\hline \multicolumn{2}{|l|}{ Treatment technique, $\mathrm{n}(\%)$} \\
\hline Coiling & $60(71.4 \%)$ \\
\hline Flow diversion & $24(28.6 \%)$ \\
\hline Complications, n (\%) & $11(13 \%)$ \\
\hline Months to the first follow up angiogram, mean (SD)* & $6.7(5)$ \\
\hline \multicolumn{2}{|l|}{ Occlusion scale on first follow up angiogram, $n(\%)^{*}$} \\
\hline Raymond 1 & $36(55.4 \%)$ \\
\hline Raymond 2 & $21(32.3 \%)$ \\
\hline Raymond 3 & $8(12.3 \%)$ \\
\hline Retreatment required, $\mathrm{n}(\%)^{* *}$ & $16(20.3 \%)$ \\
\hline Months to the final follow up angiogram, mean (SD)*** & $31.3(32.1)$ \\
\hline \multicolumn{2}{|l|}{ Occlusion scale on final follow up angiogram, $n(\%)^{* * *}$} \\
\hline Raymond 1 & $45(72.6 \%)$ \\
\hline Raymond 2 & $12(19.4 \%)$ \\
\hline Raymond 3 & $5(8.1 \%)$ \\
\hline Months to final follow up visit, mean (SD) & $23.7(29.2)$ \\
\hline mRS $0-2$ on final follow up visit, $n(\%)^{s}$ & $59(85.5 \%)$ \\
\hline Mortality before last follow up visit, $n(\%)^{\$}$ & $2(2.9 \%)$ \\
\hline
\end{tabular}

category there were 2 procedural complications (8.3\%), both of which were retroperitoneal hematomas related to femoral access.

Conclusion Endovascular treatment, and in particular, flow diversion for DACAA is safe, feasible and associated with good long-term angiographic and clinical outcomes.

Disclosures G. Porto: None. S. Al Kasab: None. M. Sattur: None. E. Almallouhi: None. O. Lajthia: None. M. Casey: None. R. Starke: None. G. Lanzino: None. C. Ogilvy: None. A. Thomas: None. L. Kim: None. R. James: None. M. Levitt: 1; C; Stryker, Medtronic. 2; C; Medtronic, Minnetronix, Metis Innovative. 6; C; Synchron, Cerebrotech. A. Spiotta: 1; C; Penumbra, Stryker, Medtronic, Terumo.

\section{E-109 PRECIPITATING HYDROPHOBIC INJECTABLE LIQUID (PHIL) IN ENDOVASCULAR TREATMENT OF BRAIN ARTERIOVENOUS MALFORMATIONS: INITIAL EXPERIENCE}

${ }^{1} \mathrm{~K}$ Orlov*${ }^{*}{ }^{1} \mathrm{~N}$ Strelnikov, ${ }^{2} \mathrm{~V}$ Berestov, ${ }^{1} \mathrm{~A}$ Somova. ${ }^{1}$ Federal Center of Brain and Neurotechnologies of Federal Medical Biological Agency of Russia, Moscow, Russian Federation; ${ }^{2}$ E.Meshalkin National Medical Research Center of Ministry of Health of Russian Federation, Novosibirsk, Russian Federation

\subsection{6/neurintsurg-2021-SNIS.204}

Background Non-adhesive embolization compositions are a method of choice in endovascular treatment of a brain arteriovenous malformation (AVM). Non-adhesive precipitating hydrophobic injectable liquid (PHIL) is a relatively new material for treating such pathologies. The objective of this paper is to demonstrate our initial experience in using of PHIL for treatment of brain AVMs. 